\title{
The Construction, Translation and Dissemination of Chinese Diplomatic Discourse
}

\author{
Ding Shi-hua \\ Xiamen University Kan Kah Kee College \\ Zhangzhou, China
}

\begin{abstract}
Translations neglecting the target audience's cognitive environment will inevitably lead to compromised acceptability of the translated texts. The optimal-relevance is what a translator should always keep in mind in the process of inference and reconstruction. Successful communication depends largely on whether a translator uses the author-intended contextual assumptions in the process of inference and makes some adaptations syntactically and socially. A translator should take relevance as his criterion, target-reader-based adaptation as his method and the author's intention as his destination to make his translated text more convergent to the original so as to present intelligible texts in line with the target audience's reading expectations.
\end{abstract}

Keywords-Diplomatic discourse; The relevance theory; Cognitive context

\section{INTRODUCTION}

Foreign publicity translation is literally translation of Chinese official speeches, work reports, etc., aimed at introducing objectively and authentically Chinese political, economic and cultural policies, achievements and developments. The primary goal of it is to promote understanding between nations, disseminate knowledge and information among all peoples. It is subject to the general principles of translation and has its own features at the same time due to its distinctive characteristics. Official materials are usually politically-sensitive issues about territorial integrity, political and military principles, and economic benefits. Any defect or mistake in its translation would be spotted or even magnified. For example, "the Chinese mainland" or "China' s mainland" is a term with implication of Chinese national sovereignty, while "mainland China" is not acceptable out of national separatism reasons. The same is true in expressions like "peaceful reunification" instead of "peaceful unification". [1] So it can be said that the quality of foreign publicity translation has great impact on foreigners' perception of a nation. It is the primary task for translators to use clear, precise and intelligible translations in communicating Chinese policies, cultures, achievements and so on to the foreign public.

However, A foreign publicity translation which is not receptor-targeted will be seriously compromised in terms of its intelligibility and acceptability among its foreign readers because any culture would prone to take in the linguistic expressions compatible to its own. The cultural compatibility requires a translator to produce translations in compliance with the social, ideological and linguistic habits of target language readers. Shen Suru suggests that it is of great importance to achieve compatibility with receptor audience by seeking commonness while elaborating the differences. Huang Youyi also proposes the principle of "Three Closeness" in foreign publicity translation: "be close to the fact of Chinese development; be close to the expected information of foreign audience; be close to the cognitive habits of foreign readers." [2]. The importance of readers' involvement in the process of translation can also be found in both Nada' $s$ equivalent translation theory and Newark's communicative translation theory.

Gut's relevance theoretic account of translation makes it more clear that translation is a three-dimensional relationship composed of source language speaker, translator and target language readers, in which the cognitive contexts and reading expectations of the target audience are taken as major concerns in the process of translation. Mechanical and rigid text-based translation with no regard to the target readers is dry and tedious (if not erroneous linguistically) to foreign audience and therefore may not achieve the ultimate goal of effective communication

\section{READER'S ROLE IN RELEVANCE TRANSLATION THEORY}

Sperber \& Wilson published in 1986 their masterpiece entitled "Relevance: Communication and Cognition", introducing for the first time the concept of relevance. An input is relevant to an individual when it connects with available contextual assumptions to yield positive cognitive effects. Human being's perceptual mechanisms quickly spot the most relevant contextual assumptions, and then make pragmatic inference with the relevant contextual assumptions. Being a decisive role in the interpretation of relevance theory, context herein is a cognition-oriented psychological construct, which is also known as contextual assumptions. It is a set of facts and assumptions which the listener is capable of representing mentally, or in others words, which are manifest to him at a given time. That is to say, all the facts and assumptions which are internalized and cognized by language users by means of experience or thinking, all the facts which are perceptible or inferable, whether true or false, contribute to an individual's total cognitive environment. Being a pivotal notion and a cognitive concept with a dynamic nature, context in this sense is not limited to information about the immediate physical environment or the immediately preceding utterances. They are 
a subset of the hearer's assumptions about the world. Expectations about the future, scientific hypotheses or religious beliefs, anecdotal memories, general cultural assumptions, beliefs about the mental state of the speaker, may all play a role in interpretation. [3]

According to the relevance theory, any communicative behavior is by nature an ostensive-inferential process. A successful communication depends on the amount of facts that are manifest to the source language author and the target language reader. The total shared cognitive environment in communication which are manifest to both parties is called mutual cognitive environment, which is the prerequisite for successful communication. Translation is essentially a doubleoperation of ostensive-inferential activity. A translator's goal is to create the mutual cognitive environment between the author and its target readers. First, a translator is expected to make correct inference to the author's communicative intention by using the author-intended contextual assumptions. Then, the translator should make sure whether the contextual assumption which the author intended to convey exist in the potential cognitive environment of the receptor audience. Generally speaking, a translator has to make correct inference to the author's communicative intention with the help of his mutual cognitive environment with the author and then provide sufficient information or facts for the target readers to identify the author's connotative intention. Relevance is of decisive importance in the process of inference and adaptation.

The relevance theory is based on two general principles: 1 the Cognitive Principle of relevance: Human cognition tends to be geared to the maximization of relevance; 2 the Communicative Principle of relevance: Every utterance (or other act of inferential communication) communicates a presumption of its own optimal relevance. [3] The major concern of the Cognitive Principle of relevance is maximum relevance, which requires the least efforts in achieving the maximum contextual effects; while the primary task of the Communicative Principle of relevance is optimal relevance, during which the speaker expects the most worthy and relevant information of processing in accordance with his cognitive abilities and preferences. The maximum relevance and the optimal relevance are totally two different notions. The readers' goal in comprehension is to find an interpretation that satisfies this expectation of optimal relevance rather than maximum relevance between the source text and his own cognitive environment. The optimal relevance should be the goal of translation, the principle and criteria for translation research, which is determined by two elements: processing effort and contextual effect. Human cognitive processes are geared to achieving the greatest possible cognitive effect with the smallest processing efforts. "Everything else being equal, the greater the positive cognitive effects achieved, the smaller the mental effort required, the greater the relevance of the input to the individual at that time."(3) It is clear to see that both processing effort and contextual effect are readers related, which require the participation of target readers in the measure of them. The optimal relevance is missing if lots of processing efforts paid by the reader can not achieve enough contextual effects. By contrast, a translation is optimal relevant if a reader can have enough or even extra contextual effects by paying moderate amount of processing efforts.

Translation is by nature an ostensive-inferential communication. It is important for a translator to make correct inference to the authors ostensive information so as to achieve the ideal contextual effects by virtue of optimal relevance pursuit. A translator should always bear in mind the author's intention and the readers' contextual expectation, figuring out their overlapped or mutual cognitive environment and making sure no unnecessary efforts are required for readers to achieve expected contextual effects. In the relevance theory, readers get involved in the whole translating process as well as serve as an important criteria for producing a successful translation. It can be said that "The relevance theoretic translation is virtually reader-oriented in which readers plays a decisive role in the success of translation [4].

\section{READERS AWARENESS IN FOREIGN PUBLICITY TRANSLATION}

Chinese Foreign publicity translation attempts to introduce to foreign readers the ideological and political stance, guiding principles and policies of Chinese government. Some political concepts are cultural-bound, some are value-laden or historically conditioned. The translation needs to be extremely precise, intelligible and readable to target language readers, which is the base line of translation. In order to communicate effectively Chinese political stand and ideas, the translator should make a careful interpretation of the political implications of the source text and manage to provide sufficient contextual assumptions to the target readers. This contextual consonance is of great significance for the seek of optimal relevance on the part of the target audience.

\section{A. Political implications of the source language}

As an important carrier for the image of a nation on the world stage, the translation strategy of political expressions will influence whether the readers of the translated language can see the real situation in China. The mechanical and simply literal translation with no regard to the contextual conflicts between the source language users and target language users will inevitably lead to the obscurity and misunderstanding on the part of the foreign readers.

For example, in the work report of the 19th National Congress of the Communist Party of China (19TH NCCPC), it is claimed that “要坚决防止的反对个人主义,分散主义, 自由 主义”. Individualism is the core idea in Western values, which promotes the exercise of one' s goals and desires and so value independence, self-reliance and individual right, opposing external interference upon one' s own interests by institutions such as the government. "Individualism" is not a derogatory term, which believes the human individual is of primary importance in the struggle for liberation and shows great respect for the value of every individual. President Xi Jinping mentioned more than once in the report the importance of individual's overall development. Official disapproval of "individualism" in the government work report will lead to the negative sentiment among western readers. Based on the 
ostensive information displayed in the report, it can be inferred that what the government really opposes is egoism and undisciplined behavior with no respect for rules and leadership. The correct translation should be "we must guard against and oppose self-centered behavior, decentralism, behavior in disregard of the rules." So it can be said that how close, how faithful, how true a version is in relation to the original, you can have nothing else in mind except the "spirit" of the original. For another instance, the work report said “我们要反对形式主 义”, which has long been translated as "we must oppose formalism". Formalism is neutral term, commonly used in the field of arts and philosophy in the west, indicating that a work's artistic value is entirely determined by its form rather than contents. The literal interpretation of "oppose formalism" would appear strange or even offensive among western readers, thinking that Chinese authority is autocratic and undemocratic.

To get fully determinate thought of the speaker, it is not enough to only deal with the denotative meaning of words. It is important to identify the illocutionary force of the utterance and achieve illocutionary force equivalence.A translator needs to make the correct inferential comprehension of the author's thought so as to figure out the implied meaning of the author. Thus, a translator has to correctly infer the author's expected intentions or the illocutionary force of the sentence with the help of identification of the optimal relevance. Failures in finding the optimal relevance will definitely lead to misinterpretation. For example: “加强基层医疗卫生服务体 系”is translated as "improve grass-root healthcare services"。 In the social context of westerners, grass-root negatively denotes the ordinary people as opposed to the leaders and authorities. The correct understanding should be "improve community-level healthcare services". For another example,

“不折腾”is a new political word frequently cited in Chinese

papers in recent years. “折腾”is a household folk expression in north China. It has three senses: 1) turn from side to side 2) do something over and over again 3) cause physical or mental suffering. Chinese former president $\mathrm{Hu}$ jintao used this expression in an important conference to reiterate the government's determination of upholding the socialist belief. It is used politically and the first accessible relevance to the author's communicative intention here is the government will firmly keep consistency in its policy. Not all the factors or assumptions available from the potential context can be elements of the actual context. People always select a context according to the principle of relevance and hope that the assumption being processed is relevant, while other less salient or non-salient factors will be automatically filtered here. It can be seen that only those factors entering the translator's "line of vision" filtered by salience of consciousness really count in translating. The less salient or even not salient elements are usually considered irrelevant and are prone to be filtered out. These choices are hierarchically ordered according to the degree of salience in the translator' s mind. [5] American news press The New Yorker failed to find the optimal relevance to the author's intention and translated into "avoid making a fuss", which was totally deviated from the author's communicative intentions. The translation from Xinhua News
Agency is "don't sway back and forth". This is the translation reflective of the author's intentions and achieves functional equivalence pragmatically.

The foreign publicity materials have always been the prime way to communicate with others and promote the positive image of a country. Translator's profound knowledge of two cultures has decisive influence on his proper linguistic choices in the process of translating. It goes without saying that the successful translation has to be contextually enriched in a variety of ways to yield a full-fledged speaker's meaning, so as to make the real and authentic China accessible to overseas readers. That is to say, having grasped the optimal relevance, the translator need to engage himself in making careful linguistic choices at different levels of the target utterance building ingredients to convey the authors' intentions by ensuring the cognitive consonance of his target audience.

\section{B. Translations that meet the aesthetic standards of target readers}

From the perspective of ideology and the values of ideas, any culture tends to actively accept and absorb the forms of expression that are compatible to it. This cultural compatibility requires the translator to have an accurate command of both cultures so as to produce acceptable translations in line with the target reader's aesthetic standards and linguistic habits. Therefore, it is necessary to make some structural adaptations on different linguistic levels in the process of translation. Adaptability is actually the property of language which enables human being to make negotiable linguistic choices from a variable range of possibilities in such a way as to approach points of satisfaction for communicative needs. [5]

Despite lots of similarities in terms of linguistic principles between the west and the east, there are many distinctive rules between the two systems when it comes to grammar and language structure. For example, Chinese and English speakers have different alignments of information, which contributes to their different syntactic structures. The structural transformation between Chinese and English boils down to the conversion from parataxis to hypotaxis. [6] Chinese is known for emphasizing parataxis and covert coherence while English stresses hypotaxis and overt cohesion. The hypotaxis characteristic of English demands explicit relationship among its constructional components by using conjunctions, relatives, participles, gerunds and infinitives to avoid semantic ambiguity or errors. As a non-inflected language, Chinese is a kind of mobile and flexible language. It doesn't have a strong formal mechanism with many connective words indicating its logical relations. These formally loose clauses need to be rearranged on the syntactic level accordingly when translated into English. It is preferable for the translator to conduct logical analysis to the Chinese source text and make it an overt-coherent expression. For example, the essence of work report of 19th NCCPC is “建设有中国特色的社会主义思想”, which is translated as "Socialism with Chinese Characteristics for a New Era". The use of "for" instead of "in" is out of the consideration that this philosophy is applied to guide the new era rather than happened in the era. 
Additionally, Chinese readers prefer repetition and fourcharacter verse styles while Western audience values conciseness and brevity. The different aesthetic standards will inevitably have an impact on their respective linguistic choices. The four-character expressions are not a rare occurrence in the work report of 19th NCCPC, which are very expressive and produce strong effect of the language. But rigid and mechanical conformity to the formal structure of these expressions will bring about syntactic confusions in the target language and strike the target readers as obscure and redundant for not giving thought to westerners' linguistic habits. For example, the report claims “绝不允许以言代法、以权压法、逐利违法、徇私枉 法”. This zero-subject imperative sentence has phonic aesthetic quality, which gives them powerful rhyming effect and sounds authoritative. English, as a hypotactic language, is compact and rigorous with its subject-predicate fixture and other adjunct components. This sentence should be reconstructed by adhering to the general subject-predicate framework of English and translated as "No one in a position of power is allowed in any way to override the law with his own orders, place his authority above the law, violate the law for personal gain, or abuse the law". The addition of the subject "no one in a position of power" helps to make the sentence structure clear and logical. So it is necessary to make a componential analysis in Chinese-English translation.

\section{Cultural Defaults in Cognitive Context}

Some Chinese political words and cultural-loaded words are initiated and commonly used in China with no equivalents in the western politics. They are expressions with distinctive Chinese characteristics and therefore do not exist in the cognitive context of Western readers. The culture default exerts great difficulties for effective and successful communication between nations. It can be said that the social-cultural context of the target cognitive environment constrains the translator's linguistic choices. As for a qualified translator, it is more important for him to have a profound and sufficient insight into two cultures instead of two languages because the senses of words are context-based in their specific culture. All the context which mirrors the social conventions and historical heritage characteristic of a certain cognitive environment, historical and cultural background, customs, religious belief, aesthetic conception, ideology and so forth, are fairly significant for the translator's practice of adaptation.

Thus, it is important for a translator to bear the socialcultural context of target readers in mind and make some linguistic adaptations in the target language. This process of adaptation is also a process of pursuing relevance. The translator should look for the optimal relevance and take a flexible approach such as literal translation, free translation, transliteration, interpretation or appropriate supplementary explanation on the basis of considering the cultural acceptability of the target language readers and observing the English language rules, so that referentially and pragmatically accurate translation can be produced. For example, the literal expression of "vegetable basket project" will strike target readers confused, thinking it is a package of policies aimed at promoting the quality of vegetables. The author and target readers do not have the contextual consonance in this regard, thus the translator needs to offer further contextual information for this cultural default. The proper translation is "a program aimed to improve the market supply of non-staple food." This is a more acceptable version yielding the contextual effects intended by the author and put the audience to no unjustifiable processing effort. For another example, "Anju Gongcheng"is a particular Chinese political term, referring to housing projects devised and constructed by authorities and sold to the medium and low income people with the cost price. This is a part of Chinese social security welfare. Chinese native readers have no problem understanding this term for their contextual consonance with the author. Whereas foreign receivers may feel confused if it is literally translated as "peaceful Living project" for their lack of the same contextual assumptions. The referent of it can not be determined until the translator reconstruct the contextual correlates here and provide some background information. So it would be better to translate it as" Affordable Housing Program", so that the target readers would not have to make excessive effort in achieving its contextual effect.

Different social and cultural context constrains the linguistic choice of a translator, who therefore should strive to bridge this gap between the author and the target readers. Ignorance of the target reader's insufficiency in cognitive context will pose enormous obstacles for their effective understanding and result in the failure of communication. Nevertheless, too much explanation will considerably reduce the readers' processing efforts and destroy the pleasure of inference. It is also important to maintain the original cultural elements and introduce alien cultures to western readers. Taking all those into consideration, a translator should take relevance as his criterion, target-reader-based adaptation as his method and the author's intention as his destination to make his translated text more convergent to the original.

\section{CONCLUSION}

Cultural globalization has increasingly highlighted the importance of foreign publicity translation. It has undoubtedly provided a good opportunity for the development of foreign publicity translation, but also raised new challenges for foreign public translation. In the translation process, the translator must think about the cognitive context of the source language and find the author-intended contextual assumptions with the help of optimal relevance, and then try to convey the communicative intentions of the author to the target readers by making adaptive translations conforming to the cultural background of foreign audiences, so that they can have the same contextual consonance with source language readers. Translators working on foreign publicity should have a keen sense of readership, strive to meet the communicative needs, interests, reading expectations, and cognitive level of the target language audience. They are supposed to use appropriate language expressions to improve the acceptability and coherence of target texts, so as to bridge the cultural gap in communication. Only by this can we truly improve the quality of foreign publicity translation and enhance the effectiveness of communication. 


\section{REFERENCES}

[1] Zheng Si-fang. Research on Foreign Publicity Translation from the Perspective of Relevance [J]. Educational Research on foreign Language \& Arts,,2013, (3). (In Chinese)

[2] Huang You-yi. The principle of "three closeness" and the resolution of difficult issues in foreign publicity translation[J].Chinese Translation, 2004, (6). (In Chinese)

[3] Sperber \& Wilson. Relevance: Communication and Cognition. [M]. Beijing: Foreign Language Teaching and Researching Press, 2001.

[4] Wu ying. A Survey of Readers' Involvement in Translation from the Perspective of Relevance Theory $[\mathrm{J}]$. Shangdong Foreign Language Teaching, 2007, (1). (In Chinese)

[5] Jef Verschueren. Understanding Pragmatics. [M]. Beijing: Foreign Language Teaching and Researching Press, 2000.

[6] Eugene Nida. Language Structure and Translation: Essays.[M]. Redwood: Stanford University Press, 1975. 